\section{European Quality of life Questionnaire}

Helga Peter ${ }^{1}$ und Thomas Penzel ${ }^{2}$

${ }^{1}$ Marburg, Deutschland

${ }^{2}$ Interdisziplinäres Schlafmedizinisches Zentrum, Charité -

Universitätsmedizin Berlin, Berlin, Deutschland

\section{Definition}

Kurzfragebogen zur Lebensqualität.

Siehe dazu $>$,Leistungs-, Schläfrigkeits- und Vigilanzmessung“‘.

\title{
Synonyme
}

EuroQol 